Article

\title{
Manifestation of Interactions of Nano-Silica in Silicone Rubber Investigated by Low-Frequency Dielectric Spectroscopy and Mechanical Tests
}

\author{
Chao Wu ${ }^{1,2}$, Yanfeng Gao ${ }^{3}$, Xidong Liang ${ }^{1, *}$, Stanislaw M. Gubanski ${ }^{1,4}{ }^{\oplus}$, Qian Wang ${ }^{1}$, \\ Weining Bao ${ }^{1}$ and Shaohua $\mathrm{Li}^{1}$ \\ 1 Department of Electrical Engineering, State Key Lab of Power System, Tsinghua University, Beijing 100084, \\ China; chaowu@uconn.edu (C.W.); stanislaw.gubanski@chalmers.se (S.M.G.); \\ qwang16@mails.tsinghua.edu.cn (Q.W.); baoweining0525@foxmail.com (W.B.); 1sh182@163.com (S.L.) \\ 2 Electrical Insulation Research Center, Institute of Materials Science, University of Connecticut, \\ Storrs, CT 06269, USA \\ 3 State Grid Jibei Electric Power Co. Ltd. Research Institute, Beijing 100045, China; gaoyfer@126.com \\ 4 High Voltage Engineering, Chalmers University of Technology, SE-412 96 Göteborg, Sweden \\ * Correspondence: lxd-dea@tsinghua.edu.cn
}

Received: 25 March 2019; Accepted: 15 April 2019; Published: 19 April 2019

\begin{abstract}
Silicone rubber composites filled with nano-silica are currently widely used as high voltage insulating materials in power transmission and substation systems. We present a systematic study on the dielectric and mechanical performance of silicone rubber filled with surface modified and unmodified fumed nano-silica. The results indicate that the different interfaces between the silicone rubber and the two types of nano-silica introduce changes in their dielectric response when electrically stressed by a sinusoidal excitation in the frequency range of $10^{-4}-1 \mathrm{~Hz}$. The responses of pure silicone rubber and the composite filled with modified silica can be characterized by a paralleled combination of Maxwell-Wagner-Sillars interface polarization and DC conduction. In contrast, the silicone rubber composite with the unmodified nano-silica exhibits a quasi-DC (Q-DC) transport process. The mechanical properties of the composites (represented by their stress-strain characteristics) reveal an improvement in the mechanical strength with increasing filler content. Moreover, the strain level of the composite with a modified filler is improved.
\end{abstract}

Keywords: silicone rubber; silica nanocomposites; interface; dielectric response; mechanical properties

\section{Introduction}

Silicone rubber composites are nowadays widely used as insulation material in power system components [1,2]. Inorganic fillers are added to polymers to improve the service performance under various operating conditions. One of the often used fillers in silicone based composites is nanometer-sized fumed silica, the addition of which helps to improve their mechanical and electrical properties. It has also been recently pointed out that the performance of a nanocomposite may deteriorate faster under long term exposure to electrical DC stress, as exemplified for nano-silica filled and unfilled crosslinked polyethylene (XLPE) [3], likely due to the changes introduced by the filler to the dynamics of charge transport in the material. A well-known phenomenon in the preparation of nanocomposite materials is the difficulty in uniformly dispersing the filler in the polymer base bulk. Nano-filler particles often tend to agglomerate, ultimately failing to exhibit the expected properties. Moreover, different physical and chemical properties of the inorganic filler and polymer matrix lead to the incompatibility in the interface. In this case the recent developments in high voltage direct current (HVDC) technology have led to new and more stringent requirements on the performance of 
insulating materials. Great efforts have thus been undertaken in surface modification of nano-filler particles to improve their dispersibility and distributivity, concurrently affecting the compatibility at particle-polymer interface, passivating surface defects and impeding moisture absorption of the fillers [4-8]. In this context, it is desirable to better understand the impact of nano-filler surface modification on the electrical transport as well as mechanical properties of composite materials for electric power applications, especially in silicone rubber-based composites. We therefore use dielectric response measurements, a powerful non-destructive tool for investigating polymer dynamics and polymer-filler interactions for elucidation of the involved charge transport behavior [4,9-18].

HVDC power systems are characterized by the existence of various voltage transient phenomena, ranging in their frequency contents from extremely low to high frequencies. They yield temporary changes of the electric field distribution within the insulation systems from the steady resistive state to a mixed capacitive-resistive state or even to purely capacitive. These variations of electric field distribution create, in turn, risk for development of breakdown events and long-term degradation. Low frequency dispersion processes, involving charge hopping in regions with weakly bonded interfaces or with signs of local degradation, have been documented at frequencies lower than $10^{-2} \mathrm{~Hz}$ [15-19]. We therefore concentrate our efforts on elucidation of charge dynamics and polarization processes in this frequency region, providing information essential for a proper design of HVDC insulation systems.

The dielectric response characteristics of silicone rubber filled with nanometer-sized silica or alumina tri-hydrate (ATH) have earlier been investigated, focusing on the impact of crosslinking agent [20], silica [21] and aluminum trihydrate (ATH) [17,18,22] contents as well as aging [23]. Our previous work revealed that the quasi-direct current (Q-DC) transport process dominates the dielectric response of ATH interface in the low frequency range [17,18]. Differing from the classical Maxwell-Wagner-Sillars interfacial polarization model [24], Q-DC process is caused by the hopping of weakly bonded charge carriers within localized paths. It can be recognized by analyzing results of dielectric response measurement in terms of the Dissado-Hill many body clusters model [13-18], wherein interactions between neighboring entities in a composite are considered. Such analyses are however still missing for silicone-silica based nanocomposites and this contribution is aiming to complete this gap. We therefore report on the effect of surface modification of nano-silica filler in silicone rubber at frequencies as low as $10^{-4} \mathrm{~Hz}$. As a reliable characterization of dielectric responses requires the use of the largest frequency range possible, this is particularly the case when low frequency dispersion processes are present. In addition, we tested the mechanical strength of the composites by measuring their stress-strain characteristics. As the mechanical strength of silicone rubber is mainly influenced by the crosslinking status [25-27], the effect of crosslinking density and distribution of crosslinking points on mechanical performance were discussed based on microstructure of the model composites.

\section{Materials and Methods}

\subsection{Material Fabrication}

Poly-methyl-vinyl silicone rubber (110-2, PMV SiR) was purchased from China Bluestar Chenggrand Co., Ltd. (Chengdu, China). The unmodified and modified fumed nano-silica (CAS 112945-52-5) were acquired from Aladin Industrial Inc., Shanghai, China and the crosslinking agent dicumyl peroxide (DCP, CAS 80-43-3) was purchased from Sigma Aldrich (St. Louis, MI, USA). Three groups of samples were prepared in this study, including pure directly vulcanized silicone rubber and silicone rubbers with 10 to 30 parts of unmodified and modified fumed silica filler per hundred parts of rubber (phr), containing $2 \mathrm{phr}$ of DCP. Thirty phr of nano-silica is a typical content providing the desired tracking and erosion resistance performance as well as mechanical properties to electrical insulation systems. The inorganic fillers and DCP were added to silicone rubber in a double-roll mixer with the nano-silica first and then the DCP. The mixing time of silica was 30 and $5 \mathrm{~min}$ for DCP. The composites were then placed in a mold and vulcanized at $443 \mathrm{~K}$ under $5 \mathrm{MPa}$ for 
$10 \mathrm{~min}$ for preparing samples with thicknesses of 1 and $2 \mathrm{~mm}$. The $1 \mathrm{~mm}$-thick samples were cut into a disc shape with a diameter of $70 \mathrm{~mm}$ for the dielectric response measurement, whereas the $2 \mathrm{~mm}$-thick ones were cut into a dumbbell shape [28] for mechanical characterization. Before each measurement, the thickness of the sample was checked by means of a micrometer at five random points and was found to vary by no more than $2 \%$. Table 1 lists the summary of the sample types, where Un_silica and Mo_silica represent the unmodified and modified silica, respectively.

Table 1. Filler content and labeling of samples used in tests.

\begin{tabular}{cccc}
\hline $\begin{array}{c}\text { Composition } \\
\text { (parts) }\end{array}$ & PMV SiR & Un_silica $x$ & Mo_silica $x$ \\
\hline $\mathrm{PMV} \mathrm{SiR} \mathrm{S}$ & 100 & 100 & 100 \\
$\mathrm{SiO}_{2}$ & 0 & $x(x=10 / 20 / 30)$ & $x(x=10 / 20 / 30)$ \\
$\mathrm{DCP}$ & 2 & 2 & 2 \\
\hline
\end{tabular}

\subsection{Parameters of Fumed Nano-Silica}

\subsubsection{Morphology}

The specific surface areas of the unmodified and modified silica fillers are 230 and $260 \mathrm{~m}^{2} / \mathrm{g}$, respectively, characterized using a Brunauer-Emmett-Teller (BET) specific surface area analyzer. The morphology of the silica filled silicone rubber composites was investigated using Transmission Electron Microscopy (TEM) (HT7700) and Scanning Electron Microscopy (SEM) (QUANTA 200 FEG). Figure 1a,b show the TEM images of nano-silica particles, indicating that the individual particle of the silica is around 10 20 nm for both kinds of silica but the particles are often clumped together into larger clusters. The clumped clusters of modified silica were around $100 \mathrm{~nm}$, whereas the agglomerates of unmodified silica were much larger, possibly forming a fractal structure [29]. Although it was proven that such big agglomerates can be dispersed when embedded in the polymer matrix [30], it can be concluded that the unmodified silica can clump together much easier than the modified silica. Figure 1c,d show the SEM images, indicating an improvement in the dispersion of the silica filler particles in the PMV SiR matrix owing to the surface modification. The white points marked in Figure $1 \mathrm{c}$ using the red arrows indicate larger agglomerates of unmodified fumed nano-silica particles. In contrast, these are not visible in the SEM images of the modified silicone rubber composite. 


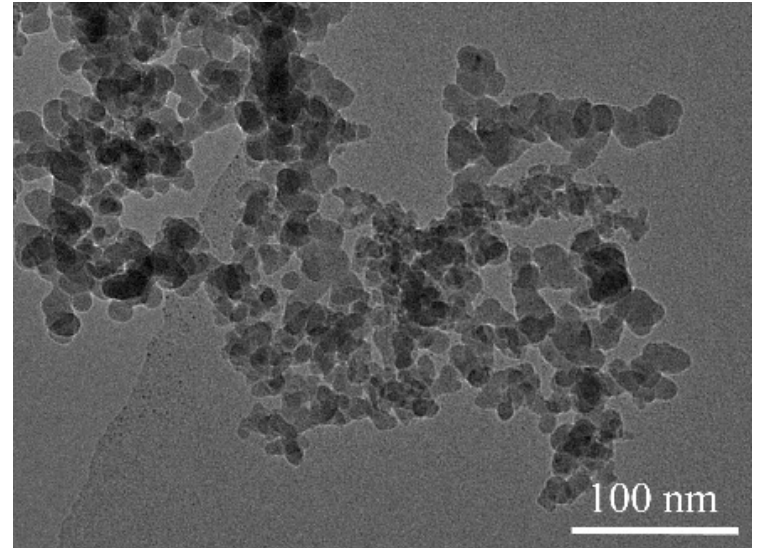

(a)

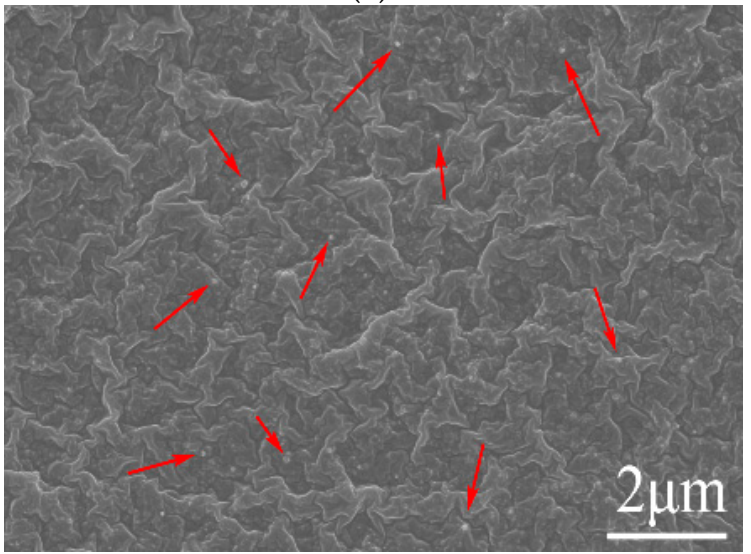

(c)

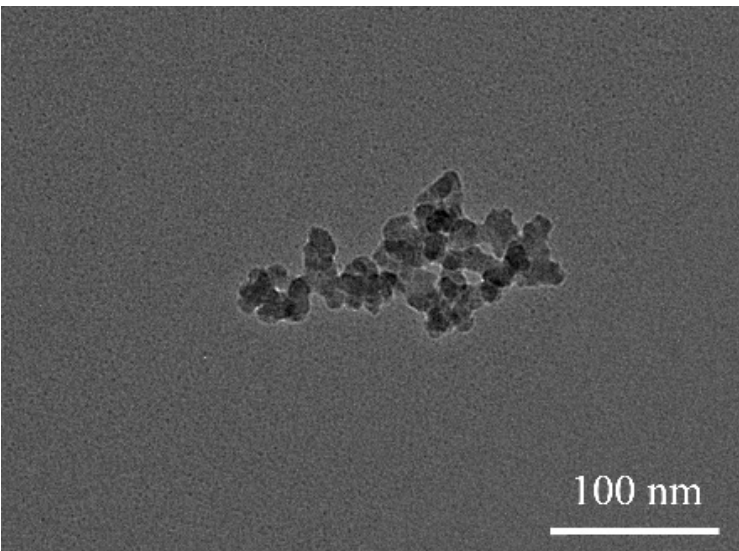

(b)

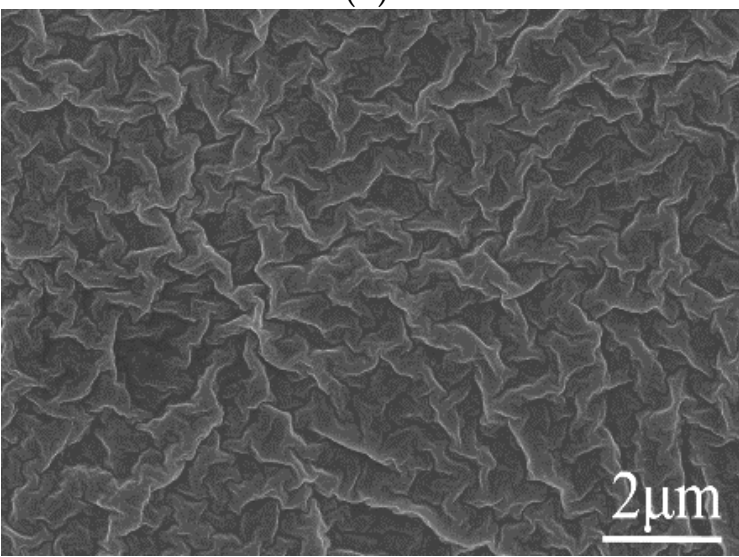

(d)

Figure 1. TEM images representing the morphology of unmodified (a) and modified (b) silica, SEM images representing the morphology of unmodified (c) and modified (d) silica filled silicone rubber composites.

\subsubsection{FTIR and XPS Analyses}

A Fourier Transform Infrared Spectrometer (FTIR) was used to investigate the chemical groups present on the surfaces of the nano-silica particles. The results are illustrated in Figure 2. The three peaks at 2855,2929 , and $2957 \mathrm{~cm}^{-1}$ of the transmittance spectrum of the modified nano-silica fillers represent the stretching vibration of $\mathrm{C}-\mathrm{H}$ groups, indicating the existence of $-\mathrm{CH}_{2}$ and $-\mathrm{CH}_{3}$ groups on their surface, introduced by the surface modification agent. The wide peak at $3420 \mathrm{~cm}^{-1}$ reveals the existence of hydroxyl groups on the surfaces of both types of fillers whereas the small peak at $1631 \mathrm{~cm}^{-1}$ represents the bending vibration of the hydroxyl groups. The broad peak in the range of $1000-1200 \mathrm{~cm}^{-1}$ and at $810 \mathrm{~cm}^{-1}$ is the stretching vibration of $\mathrm{Si}-\mathrm{O}$ bond.

Figure 3 shows the chemical elements present on the surface of the nano-silica filler, revealed by X-ray photoelectron spectroscopy (XPS). The pronounced higher intensity of the C(1s) peak of the modified nano-silica fillers confirms the existence of carbon chains introduced by the surface modification. As a commercially available nano-silica was used, FTIR and XPS analyses confirmed that carbon chains dominate on the modified surface of the filler particles and there are no other elements present in addition to silicon and oxygen. 


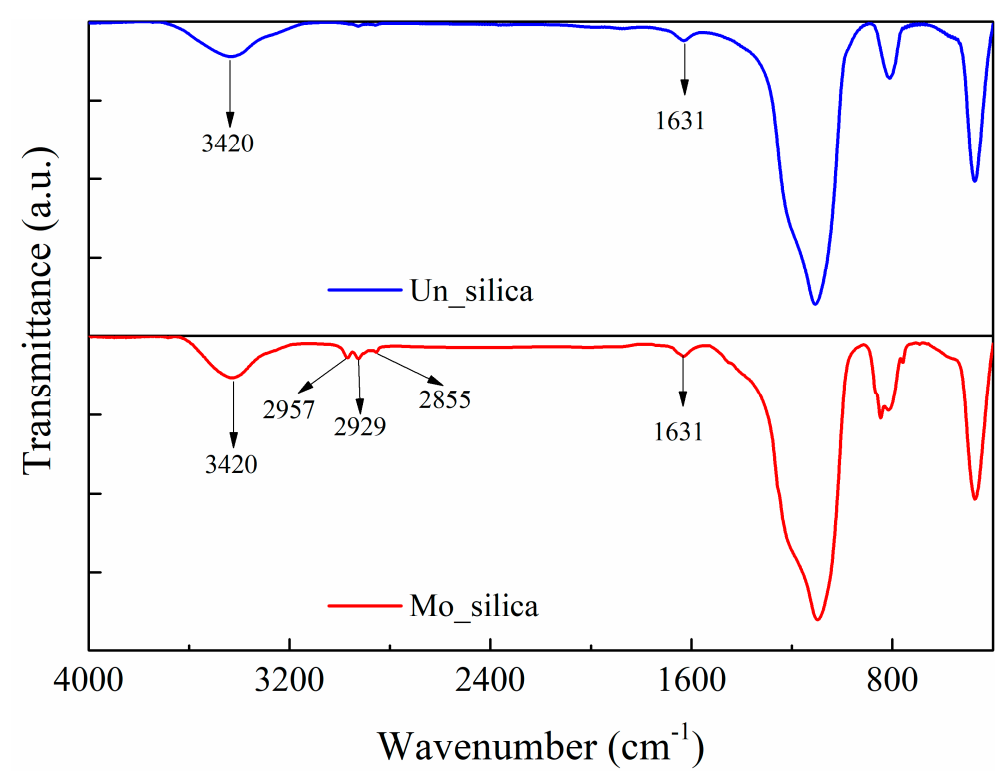

Figure 2. Chemical groups on the surface of unmodified and modified fumed nano-silica fillers investigated by FTIR.

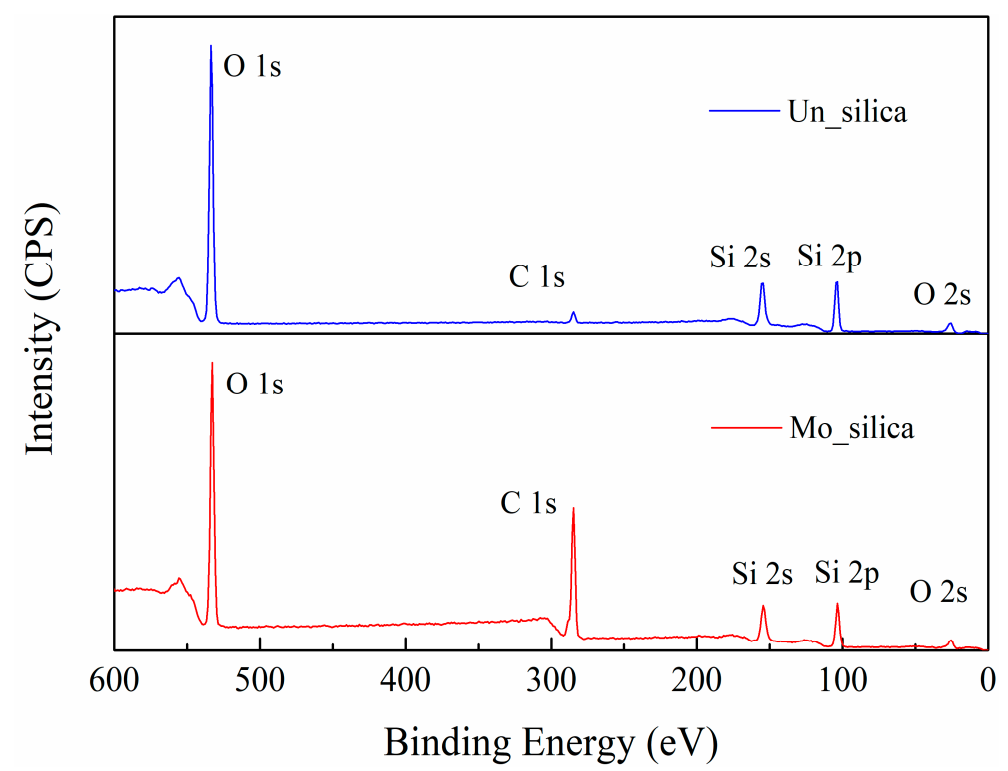

Figure 3. Chemical elements on the surface of unmodified and modified fumed nano-silica fillers obtained by XPS.

\subsection{Dielectric Response Measurement}

A chamber with the sample and electrode was kept in an oven which allowed the limiting of temperature fluctuation within $\pm 0.5 \mathrm{~K}$ throughout the duration of the measurement. The measuring AC voltage level was set at $2.0 \mathrm{Vrms}$, and the frequency was varied between $10^{-4}$ to $1 \mathrm{~Hz}$ with seven points per decade. The measurement was conducted under three temperatures of 293,323 and $353 \mathrm{~K}$, which are much higher than the glass transition temperature $\left(T_{\mathrm{g}}\right)$ of around $230 \mathrm{~K}$ for the silicone rubber composites.

A seven-hour delay before the measurement was set to ensure that the temperature of the sample was the same as the set measurement temperature. At each measurement point, the data was acquired after two voltage cycles. This allows a reliable characterization of the dielectric response and is consistent with the limitations of instrument accuracy. Before the measurements, the samples were cleaned with ethanol and deionized water to remove impurities from their surface. To avoid the 
presence of water during the measurements, the samples were additionally kept in an oven at $363 \mathrm{~K}$ for $10 \mathrm{~h}$ before the measurements. In order to ensure that no effect of aging had occurred during the measurement, a preliminary check was made by repeating the measurements at the highest temperature and no differences between the measurements were observed.

\subsection{Mechanical Strength Measurement}

The stress-strain characteristics of the investigated materials were obtained using a tensile testing machine (Zwick Z2.5) under a stretching rate of $500 \mathrm{~mm} / \mathrm{min}$. The dumbbell-shaped samples were prepared according to the standard ISO 37: 2005(E) [28], type 1A sample.

\section{Results}

\subsection{Capacitance and Susceptibility}

Figure 4 shows the complex capacitance $C^{*}(\omega)$ and susceptibility $\chi^{*}(\omega)$ of pure silicone rubber (PMV SiR) and silicone rubber with nano-silica in logarithmic coordinates with solid and open dots representing their real and imaginary parts, respectively. $\chi^{*}(\omega)$ was calculated from the measured complex capacitance $C^{*}(\omega)$ using Equation (1), where $\varepsilon^{\prime}(\omega)$ and $\varepsilon^{\prime \prime}(\omega)$ denote the real and imaginary components of the relative permittivity, respectively. The infinite frequency term $\varepsilon(\infty)$ represents the contribution of the dielectric response in high frequency region beyond the measurement window [31].

$$
C^{*}(\omega)=C^{\prime}(\omega)-i C^{\prime \prime}(\omega)=\varepsilon_{0} \frac{A}{d}\left[\varepsilon^{\prime}(\omega)-i \varepsilon^{\prime \prime}(\omega)\right]=\varepsilon_{0} \frac{A}{d}\left[\chi^{\prime}(\omega)-i \chi^{\prime \prime}(\omega)+\varepsilon(\infty)\right]=\varepsilon_{0} \frac{A}{d}\left[\chi^{*}(\omega)+\varepsilon(\infty)\right]
$$

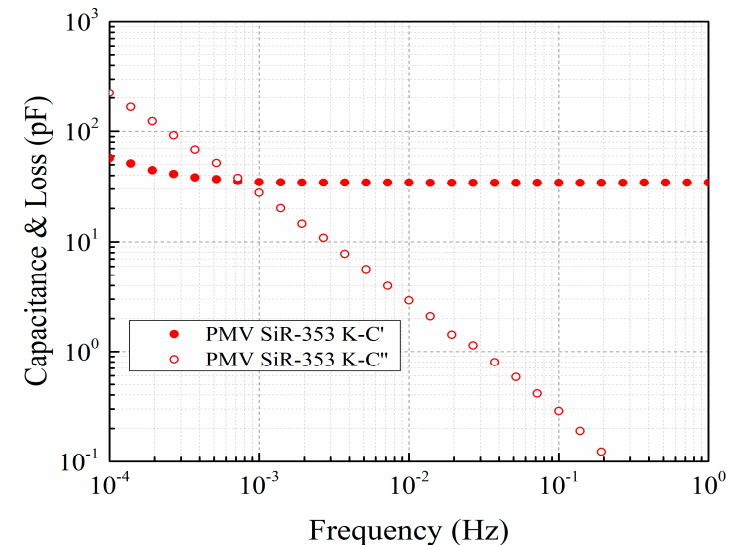

(a)

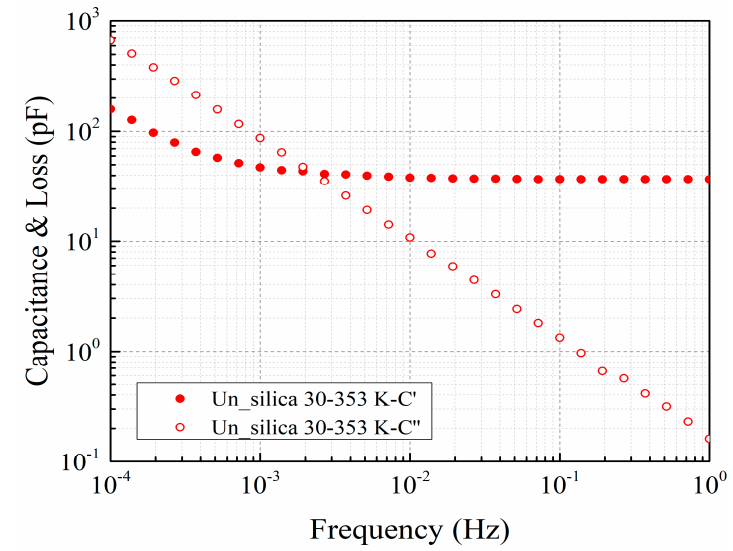

(c)

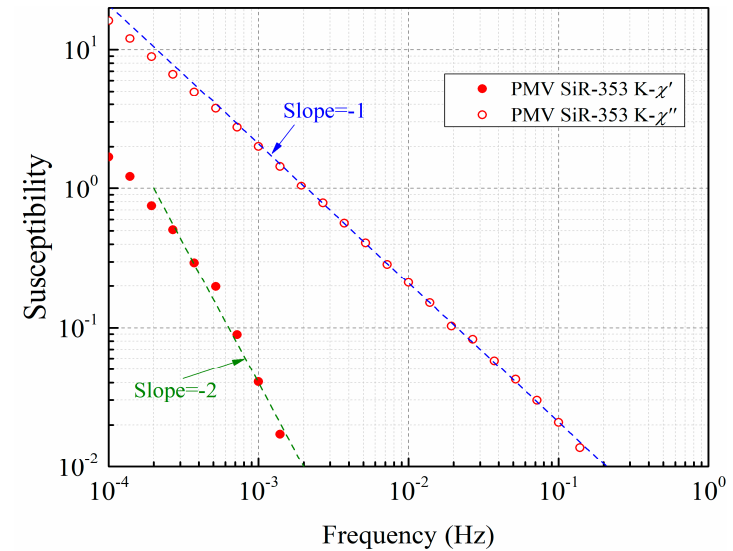

(b)

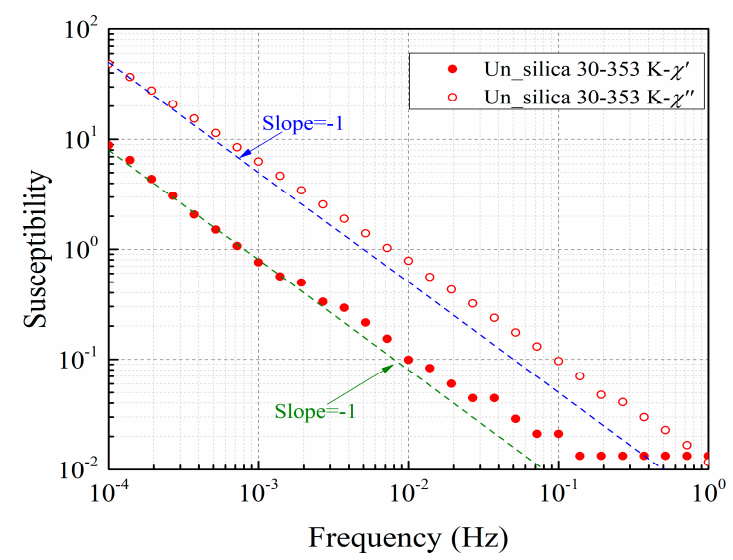

(d)

Figure 4. Cont. 


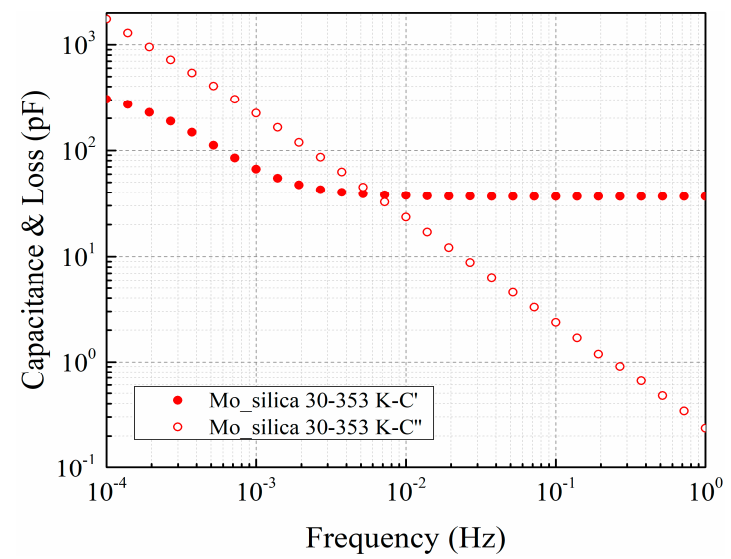

(e)

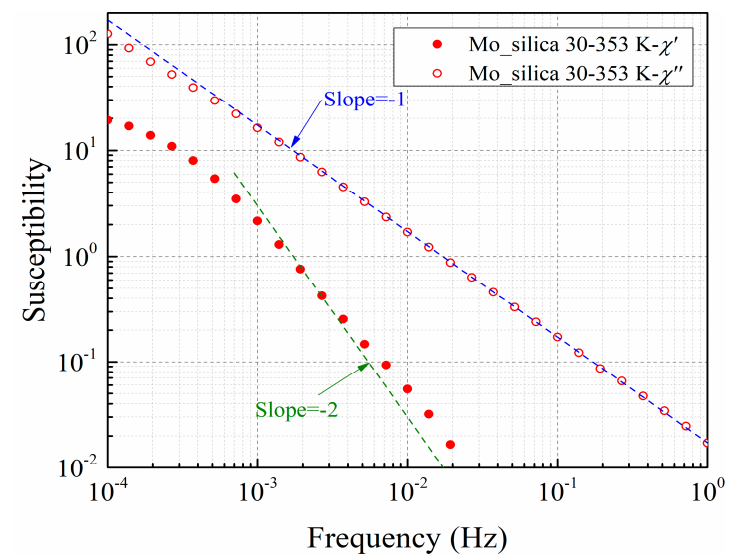

(f)

Figure 4. Frequency dependence of complex capacitance and susceptibility of pure silicone rubber (a) and (b), silicone rubber with unmodified nano-silica (c) and (d), silicone rubber with modified nano-silica (e) and (f).

The figures show that the results for pure silicone rubber and silicone rubber with surface modified nano-silica are similar among the three types of samples. In the double logarithm coordinate, trend lines with slopes of -1 and -2 are marked in the $\chi^{*}(\omega)$ graphs. In the $\chi^{\prime \prime}(\omega)$ curves of pure silicone rubber and silicone rubber filled with modified nano-silica, except for the deviation at a frequency approaching $10^{-4} \mathrm{~Hz}$, the data points coincide well with the trend line of slope -1 . For $\chi^{\prime}(\omega)$, the variation trend has a slope approaching -2 . In contrast, for the silicone rubber filled with unmodified nano-silica, the shape of $\chi^{*}(\omega)$ is quite different. The slope of $\chi^{\prime \prime}(\omega)$ is greater than -1 and is parallel with the slope of $\chi^{\prime}(\omega)$, which is a characteristic of the Q-DC process in the Dissado-Hill clusters model. In the MWS models, the frequency dependence of the real part of the susceptibility should remain saturate with the decrease of frequency.

\subsection{Equivalent Circuit Analysis}

More than one type of dielectric relaxation process is included in the silicone rubber composites. The $C^{*}(\omega)$ correlated to individual dielectric processes can be represented as in parallel or in series connected equivalent circuit components, depending on the morphology and composition of the sample.

Figure 5a shows the Q-DC conductance process for silicone rubber filled with unmodified nano-silica. In the cases of pure silicone rubber and silicone rubber filled with modified nano-silica, the DC conductance and MWS interface polarization process are included as parallel branches in the equivalent circuit, shown in Figure 5b.

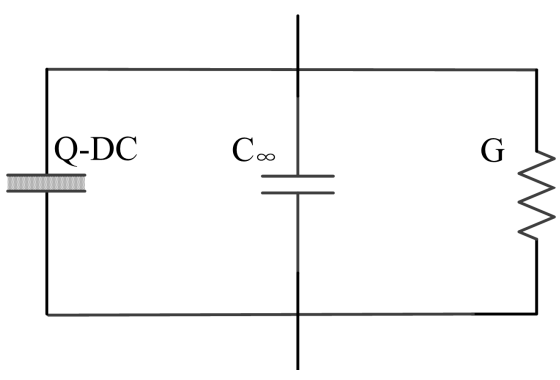

(a)

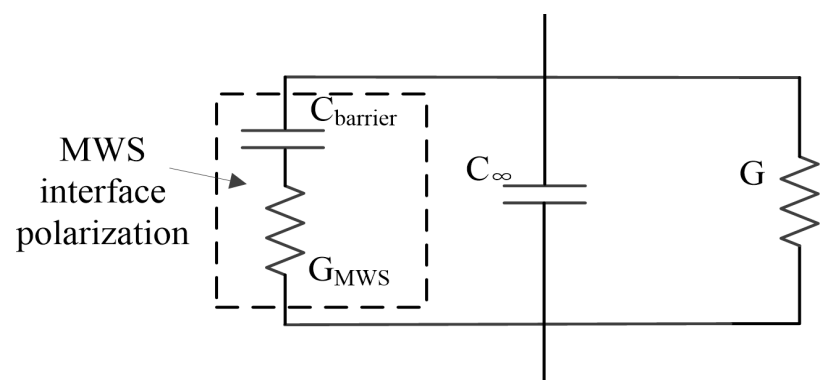

(b)

Figure 5. Equivalent circuits of silicone rubber filled with unmodified nano-silica (a). Equivalent circuits of pure silicone rubber and silicone rubber filled with modified fumed nano-silica (b). 
The functional dependences of $C^{*}(\omega)$ and $\chi^{*}(\omega)$ on the MWS polarization can be characterized using Equations (2) and (3).

$$
\begin{gathered}
C^{*}(\omega)=C_{\text {barrier }} \frac{1}{1+i \omega \tau}=C_{\text {barrier }} \frac{1-i \omega \tau}{1+\omega^{2} \tau^{2}} ;\left(\tau=\frac{C_{\text {barrier }}}{G_{\mathrm{MWS}}}\right) \\
\chi^{*}(\omega)=\chi_{0} \frac{1}{1+i \omega \tau}=\chi_{0} \frac{1-i \omega \tau}{1+\omega^{2} \tau^{2}} ;\left(\tau=\frac{C_{\text {barrier }}}{G_{\mathrm{MWS}}}\right)
\end{gathered}
$$

In the Dissado-Hill clusters Q-DC model, the weak bonded charge pairs can be separated but remain not as absolutely free as in the DC conduction process. Thus, the susceptibility in the Q-DC process follows Equation (4) [19]:

$$
\chi^{*}(\omega)=\chi_{0} \frac{\Gamma(1-p-n)}{\Gamma(1-n) \Gamma(1-p)}\left(1+i \frac{\omega}{\omega_{c}}\right)^{n-1} \times{ }_{2} F_{1}\left(1-n, 1+p ; 2-n ;\left(1+i \frac{\omega}{\omega_{c}}\right)^{-1}\right)
$$

Here, $\Gamma(\mathrm{a})$ is the gamma function and ${ }_{2} F_{1}(\mathrm{a}, \mathrm{b} ; \mathrm{c} ; \mathrm{z})$ is the Gaussian hypergeometric function. $\chi_{0}$ denotes the net concentration of the charge carriers produced by a unit electric field, and $\omega_{c}$ is the characteristic angular frequency. At angular frequencies greater than $\omega_{c}$, the weakly bonded charge pairs move as a coupled dipole over a cluster of sites. At lower frequencies, the charges are so weakly bonded to their counter charges that they can transfer between different clusters. The exponents $p$ and $n(0<p, n<1)$ characterize the inter-cluster and inner-cluster interactions of the charge carriers, respectively. The index $p$ defines the dynamic connection of the charge transport between clusters, whereas the index $n$ represents the interactions of charge-pair motion within the clusters. The susceptibility in the Q-DC process can be thus characterized by two power law like responses as follows.

$$
\begin{aligned}
& \chi^{\prime \prime}(\omega) \propto \chi^{\prime}(\omega) \propto \omega^{n-1}, \omega>>\omega_{c} \\
& \chi^{\prime \prime}(\omega) \propto \chi^{\prime}(\omega) \propto \omega^{-p}, \omega<<\omega_{c}
\end{aligned}
$$

It should be noted that at lower and higher frequency ranges, $x^{\prime}$ and $x^{\prime \prime}$ become parallel to each other in the $\log x-\log \omega$ coordinates, and therefore, the Q-DC response is also called a constant phase angle (CPA) response [19].

Figure 6 shows the fittings of the experimental results of the measured complex capacitance with the model circuits for silicone rubber filled with unmodified and modified nano-silica fillers. Tables 2 and 3 list the employed equivalent circuit parameters representing the resolved terms for complex capacitance of Q-DC, MWS interface polarization, DC conductance, and geometric capacitance.

\begin{tabular}{|c|c|c|}
\hline \multicolumn{2}{|c|}{ Circuit Element } & \multirow{2}{*}{$\begin{array}{c}\text { Parameters } \\
8.58 \times 10^{-4} \\
>50 \times 2 \pi \\
0.9 \\
-\end{array}$} \\
\hline $\begin{array}{c}\text { Q-DC } \\
\text { process }\end{array}$ & $\begin{array}{c}\chi(0) \\
\omega_{\mathrm{c}}(\mathrm{rad} / \mathrm{s}) \\
\mathrm{p} \\
\mathrm{n}\end{array}$ & \\
\hline \multicolumn{2}{|c|}{$\mathrm{G}(\mathrm{pS})$} & $9.15 \times 10^{-5}$ \\
\hline \multicolumn{2}{|c|}{$\mathrm{C}_{\infty}(\mathrm{pF})$} & 36.79 \\
\hline
\end{tabular}
Limited by the data, we can only obtain the parameters related to the lower frequency branch of the Q-DC process. Nevertheless, it clearly reveals the difference between the responses of the silicone rubbers filled with unmodified and modified nano-silica.

Table 2. Parameters of electric circuit elements for silicone rubber filled with unmodified nano-silica. 
Table 3. Parameters of electric circuit elements for silicone rubber filled with modified nano-silica.

\begin{tabular}{|c|c|c|}
\hline \multicolumn{2}{|c|}{ Circuit Element } & Parameters \\
\hline \multirow{4}{*}{$\begin{array}{c}\text { MWS } \\
\text { polarization }\end{array}$} & $\mathrm{C}_{\text {barrier }}(\mathrm{pF})$ & 265.3 \\
\hline & $\mathrm{G}_{\mathrm{MWS}}(\mathrm{pS})$ & 0.55 \\
\hline & $\chi(0)$ & 19.2 \\
\hline & $\omega_{\mathrm{mws}}(\mathrm{rad} / \mathrm{s})$ & $1.3 \times 10^{-2}$ \\
\hline \multirow{2}{*}{\multicolumn{2}{|c|}{$\begin{array}{l}\mathrm{G}_{\mathrm{dc}}(\mathrm{pS}) \\
\mathrm{C}_{\infty}(\mathrm{pF})\end{array}$}} & 0.986 \\
\hline & & 37.2 \\
\hline
\end{tabular}

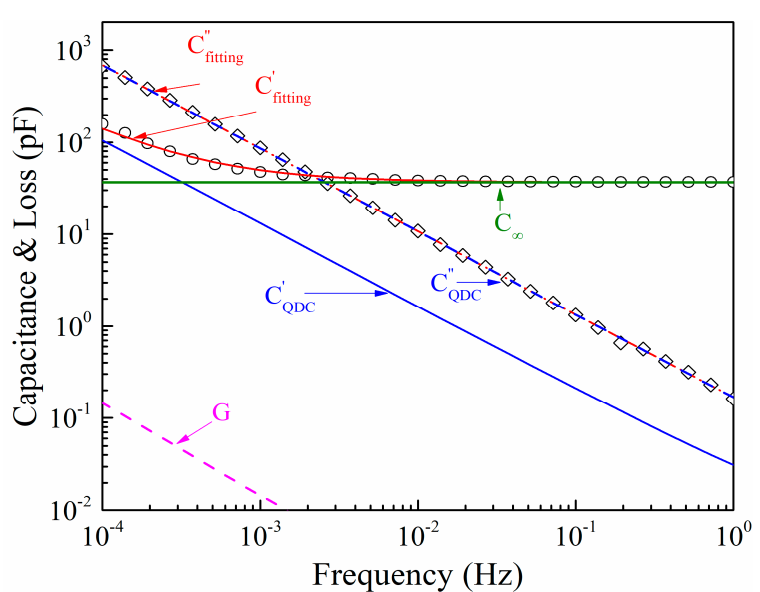

(a)

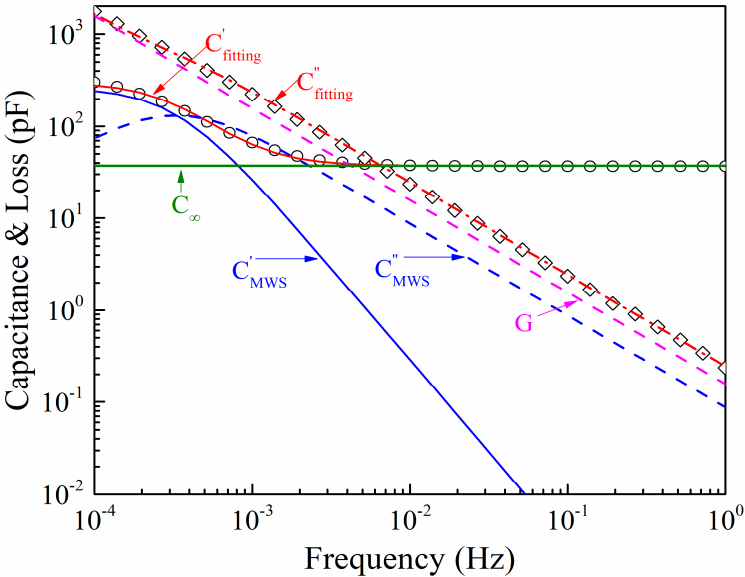

(b)

Figure 6. Equivalent circuit fittings for silicone rubber filled with unmodified fumed nano-silica (a) and modified fumed nano-silica (b) at $353 \mathrm{~K}$.

\subsection{Temperature Dependence of Susceptibility}

One of the advantages of temperature normalization of dielectric response measurement data is the improved reliability with the increase in the observation frequency range [15-18,32,33]. To confirm the characteristics discussed above, the dielectric response measurements were repeated at 293 and $323 \mathrm{~K}$. The obtained data at 293 and $323 \mathrm{~K}$ are thereafter shifted into the position of data at $353 \mathrm{~K}$, forming so called master curves, as shown in Figure 7. The data at different temperatures for both types of the sample coincide well, indicating that the operating response mechanisms remain the same in the analyzed temperature range.

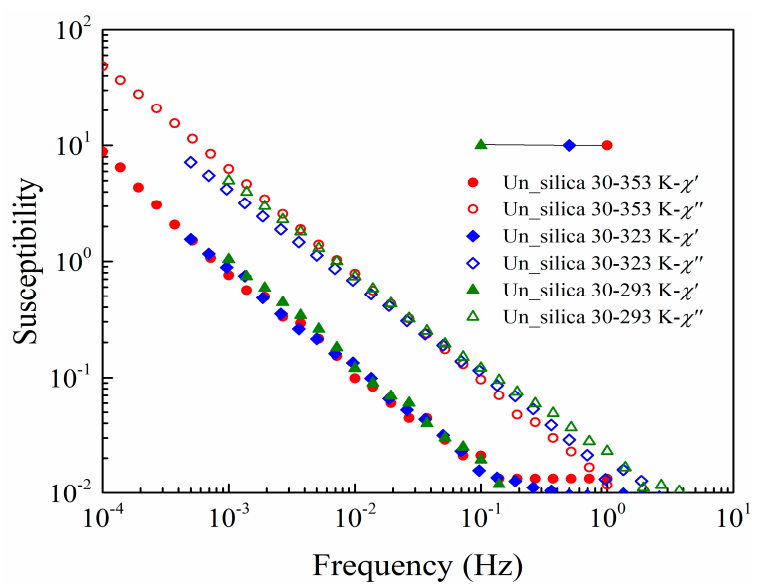

(a)

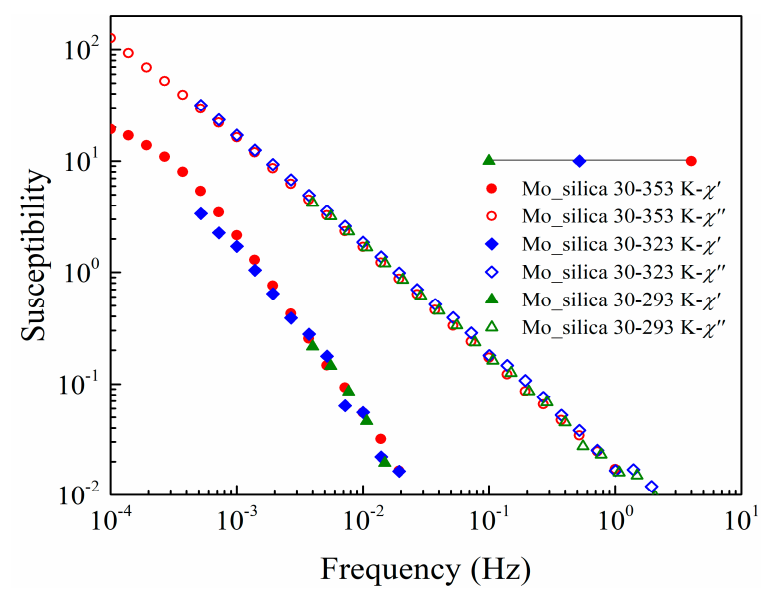

(b)

Figure 7. Master curves of silicone rubber composites filled with unmodified (a) and modified fumed nano-silica (b) at different temperature. 


\subsection{Mechanical Performance}

Figure 8 shows the stress-strain characteristics of the investigated composites. In Figure $8 \mathrm{~b}$, data points, with content of silica being zero, reveal the tensile strength and elongation at the break of the silicone rubber without nano-silica filler. The tensile strength and elongation at the break improve significantly with the addition of fumed nano-silica into the composite system. The comparison of the characteristics of silicone rubbers filled with different contents of unmodified and modified nano-silica shows that the tensile strength remains similar for the unmodified and modified nano-silica composites at the same filler content. However, the elongation at the break becomes significantly greater for the modified fumed nano-silica composites. As the mechanical properties of cross-linked rubbers are mainly influenced by the crosslinking density [27], the bonds formed between the nano-silica filler and the polymer matrix can be treated as physical cross-linking points [25]. In addition, the organic chains on the surface of modified nano-silica can react with the polymer chains of the silicone rubber matrix in the cross-linking reaction. Thus, the increased effective cross-linking density results in higher mechanical strength. The surface properties and dispersion degree of nano-silica can also influence the physical cross-linking effect. A poor dispersion and uneven distribution of the filler particles lead to only localized physical cross-linking regions. The stress will accumulate in these region compared to the polymer matrix regions. In contrast, a good dispersion and distribution of the filler provides a relatively uniform cross-linking, distributing the applied stress in a way that the polymer chains in the network bear similar stress loads [27]. The stress concentration will, on the other hand, result in a break at lower elongations. However, an increasing content of nano-silica increases the possibility of agglomeration, making the observed difference in the elongation at break for samples with 30 parts of nano-silica obvious.

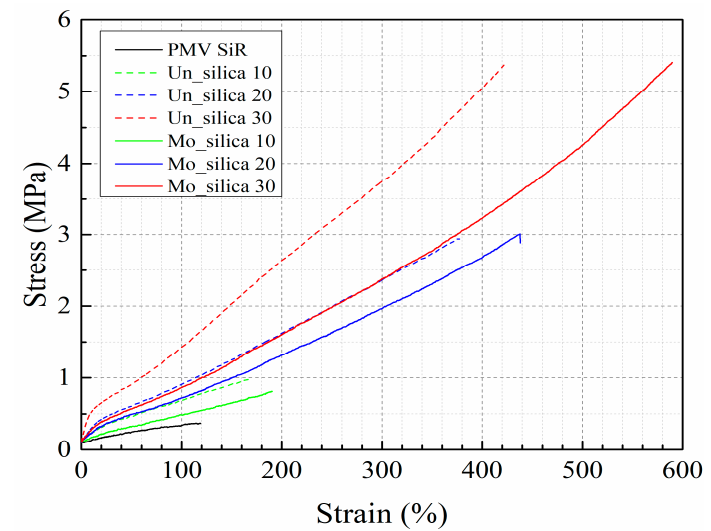

(a)

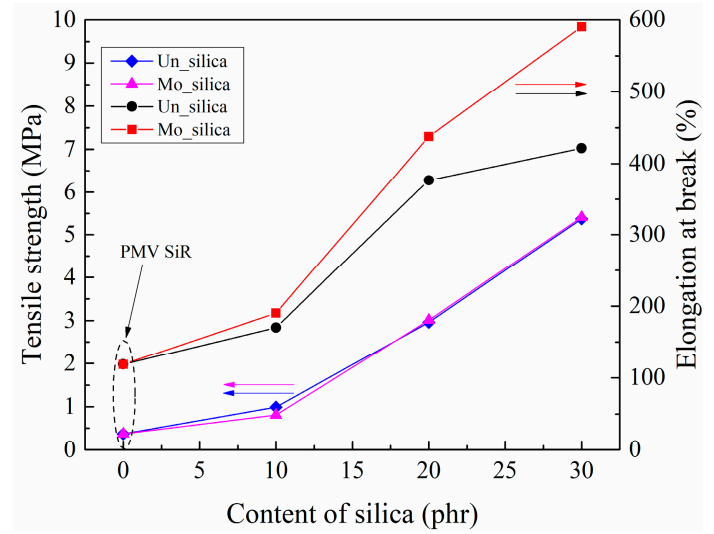

(b)

Figure 8. Tensile stress-strain curves (a) and mechanical strength (b) of silicone rubber composites filled with unmodified and modified nano-silica filler.

\section{Discussion}

The surface modification by the coupling agent improves the dispersion degree of nano-silica in silicone rubber, which implies greater inter-particle distances. The characteristics of charge transport become similar to those observed in pure silicone rubber, i.e., dominated by the real DC conduction process. In contrast, for the unmodified nano-silica filled rubber, the greater agglomeration of the filler particles and the likely formation of a polymer interphase between them [34], which is presented as the green region around the particles in Figure 9, lead to a Q-DC transport process, wherein the local overlap of the interface provides channels for a localized movement of the charges, as observed in elsewhere [35]. The difference between the behaviors of silicone rubber filled with unmodified and modified nano-silica can be comprehended as follows. The charge transport through the polymer matrix away from the filler particles and the bonded interfacial regions allow a free charge transport process, wherein the motions of single charges do not affect each other. In the agglomerate groups, with overlapping interfacial 
regions, this motion does not become entirely free because of the interactions with the charges located in the neighboring positions. Such charges can form larger cluster dipoles, the dynamics of which form the Q-DC response.
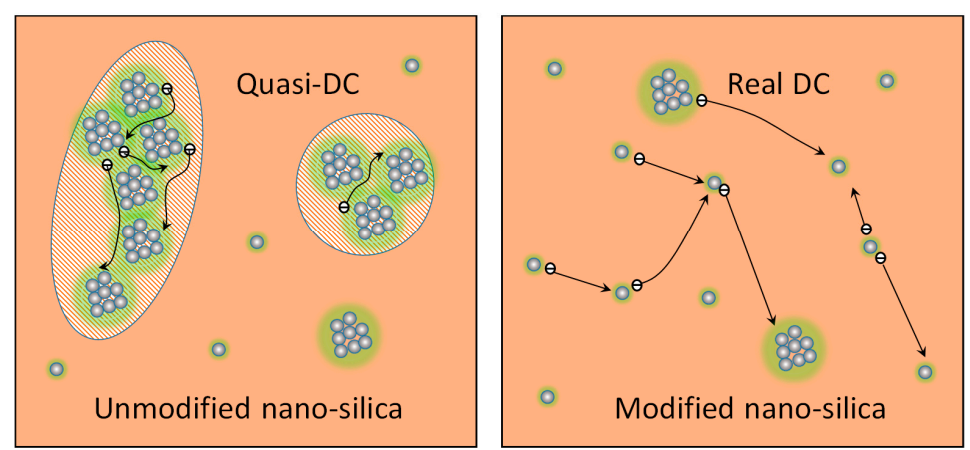

Figure 9. Schematic views of charge interactions in unmodified and modified silica filled silicone rubber composites.

With regard to the chemical interaction between silicone rubber matrix and nano-silica, the hydroxyl groups present on both types of the filler (broad FTIR peak at $3420 \mathrm{~cm}^{-1}$ ) form hydrogen bonds with oxygen atoms in the siloxane backbone. Moreover, for the modified nano-silica, the interactions between the organic chains in the surface treated nano-silica and the silicone rubber matrix are stronger because of the better compatibility of the organic phases. According to the polymer chain alignment model of [34], a layer of the aligned polymer chains introduced by the surface modification can penetrate into the host polymers and interact with the base polymer chains, forming an interpenetrating network with no free positions for charges hopping. The charges accumulated at the interface are thus transported through the rubber matrix (DC conductance process). In contrast, for the unmodified filler, the weaker interactions between silicone rubber and silica combined with the effect of agglomeration can provide free positions and pathways for charge hopping.

In terms of the mechanical performance, a better dispersion of the physical cross-linking points lead to a more uniform stress distribution in the composite system. On the other hand, the agglomeration of the nano-silica filler particles and the resulting lack of physical crosslinking within the agglomerates contribute toward stress accumulation. In addition, the agglomeration restricts the deformation of the polymer chains [25]. As in the stretching process, the motion of the polymer chains leads to a change in the relative position of the cross-linking points (of both the chemical and the physical ones); it is more difficult to introduce this change in the agglomeration clusters [30]. Moreover, the packed polymer chains in the clusters of crosslinking points yield a steeper slope of the stress-strain curve [27]. We thus conclude that the higher Young's modulus of the unmodified nano-silica filled silicone rubber composite is a result of the stress accumulation and the reduction in the deformation possibility, but not of the increased cross-linking density.

\section{Conclusions}

The results of dielectric response measurements conducted at low frequency range from $10^{-4}$ to $1 \mathrm{~Hz}$ clearly show how the surface modification of nano-silica filler influences the electric transport mechanism in silicone rubber composites. The charge transport process in modified fumed nano-silica filled silicone rubber composite became similar to that in pure silicone rubber matrix and it followed the DC conduction behavior. In contrast, the composite filled with unmodified nano-silica revealed the operation of the quasi-DC process, in which the frequency dependence of the dielectric susceptibility follows the power law dependence. The agglomeration and thus the overlapping of the interface regions of the neighboring particles in the clusters form local hopping centers, thereby allowing local charge interactions that manifest in the form of a Q-DC process, as proposed in the Dissado-Hill clusters model. 
The stress-strain characteristics of the studied silicone rubber composites show a significant improvement in their mechanical strength owing to the formation of additional physical cross-linking points. The improved filler particle dispersion in the surface modified nano-silica composite led to a uniform stress distribution during stretching, yielding a greater elongation at break. However, the tensile strength remained similar for both types of the composites at the same filler content, indicating that the adhesive strength between the polymer chains and the nano-silica particles was not strongly affected. As for the unmodified filler, the agglomeration restricted the deformation of the polymer chains and increased the difficulty in changing the relative position of the filler particles, thereby increasing the Young's modulus of the composite.

Author Contributions: C.W. fabricated the material samples and conducted the dielectric response measurements. Y.G. contributed to the analysis and interpretation of dielectric response. Q.W., W.B. and S.L. performed the morphology, FTIR and XPS characterization. C.W. prepared the original draft and S.M.G. contributed to the analyses and editing. X.L. proposed the concept and supervised the whole work.

Acknowledgments: Financial support from the National Key Research Program of China (No. 2016YFB09008) is gratefully acknowledged. The authors wish to thank the High Voltage Engineering laboratory of the University of Leicester, UK, for the support in dielectric response tests.

Conflicts of Interest: The authors declare no conflict of interest.

\section{References}

1. Liang, X.; Wang, S.; Fan, J.; Guan, Z. Development of composite insulators in China. IEEE Trans. Dielectr. Electr. Insul. 1999, 6, 586-594. [CrossRef]

2. Gubanski, S.M. Modern outdoor insulation-concerns and challenges. IEEE Electr. Insul. Mag. 2005, 21, 5-11. [CrossRef]

3. Lei, W.; Wu, K.; Wang, Y.; Cheng, Y.; Zheng, X.; Dissado, L.A.; Dodd, S.J.; Chalashkanov, N.M.; Fotherglll, J.C.; Zhang, C.; et al. Are nano-composites really better DC insulators? A study using silica nanoparticles in XLPE. IEEE Trans. Dielectr. Electr. Insul. 2017, 24, 2268-2270. [CrossRef]

4. Marx, P.; Wanner, A.; Zhang, Z.; Jin, H.; Tsekmes, I.; Smit, J.; Kern, W.; Wiesbrock, F. Effect of interfacial polarization and water absorption on the dielectric properties of epoxy-nanocomposites. Polymers 2017, 9, 195. [CrossRef] [PubMed]

5. Liu, J.; Cheng, Y.; Xu, K.; An, L.; Su, Y.; Li, X.; Zhang, Z. Effect of nano-silica filler on microstructure and mechanical properties of polydimethylsiloxane-based nanocomposites prepared by "inhibition-grafting" method. Compos. Sci. Technol. 2018, 167, 355-363. [CrossRef]

6. Li, H.; Liu, F.; Tian, H.; Wang, C.; Guo, Z.; Liu, P.; Peng, Z.; Wang, Q. Synergetic enhancement of mechanical and electrical strength in epoxy/silica nanocomposites via chemically-bonded interface. Compos. Sci. Technol. 2018, 167, 539-546. [CrossRef]

7. Zhang, L.; Khani, M.M.; Krentz, T.M.; Huang, Y.; Zhou, Y.; Benicewicz, B.C.; Nelson, J.K.; Schadler, L.S. Suppression of space charge in crosslinked polyethylene filled with poly(stearyl methacrylate)-grafted $\mathrm{SiO}_{2}$ nanoparticles. Appl. Phys. Lett. 2017, 110, 132903. [CrossRef]

8. Pourrahimi, A.M.; Pallon, L.K.H.; Liu, D.; Hoang, T.A.; Gubanski, S.; Hedenqvist, M.S.; Olsson, R.T.; Gedde, U.W. Polyethylene Nanocomposites for the Next Generation of Ultralow-Transmission-Loss HVDC Cables: Insulation Containing Moisture-Resistant MgO Nanoparticles. ACS Appl. Mater. Interfaces 2016, 8, 14824-14835. [CrossRef]

9. Otegui, J.; Schwartz, G.A.; Cerveny, S.; Colmenero, J. Influence of water and filler content on the dielectric response of silica-filled rubber compounds. Macromolecules 2013, 46, 2407-2416. [CrossRef]

10. Min, D.; Yan, C.; Huang, Y.; Li, S.; Ohki, Y. Dielectric and carrier transport properties of silicone rubber degraded by gamma irradiation. Polymers 2017, 9, 533. [CrossRef]

11. Linde, E.; Verardi, L.; Fabiani, D.; Gedde, U.W. Dielectric spectroscopy as a condition monitoring technique for cable insulation based on crosslinked polyethylene. Polym. Test. 2015, 44, 135-142. [CrossRef]

12. Kremer, F.; Schönhals, A. Broadband Dielectric Spectroscopy; Springer: Berlin, Germany, 2003.

13. Dissado, L.A.; Rowe, R.C.; Haidar, A.; Hill, R.M. The Characterization of Heterogeneous Gels by Means of a Dielectric Technique I. Theory and Preliminary Evaluation. J. Colloid Interface Sci. 1987, 117, 310-324. [CrossRef] 
14. Rowe, R.C.; Dissado, L.A.; Zaidi, S.H.; Hill, R.M. The Characterization of Heterogeneous Gels by Means of a Dielectric Technique II. Formulation and Structural Considerations. J. Colloid Interface Sci. 1998, 122, 354-366. [CrossRef]

15. Abdelmalik, A.A.; Dodd, S.J.; Dissado, L.A.; Chalashkanov, N.M.; Fothergill, J.C. Charge transport in thermally aged paper impregnated with natural ester oil. IEEE Trans. Dielectr. Electr. Insul. 2014, 21, 2318-2328. [CrossRef]

16. Chalashkanov, N.M.; Dodd, S.J.; Dissado, L.A.; Fothergill, J.C. Re-examination of the Dielectric Spectra of Epoxy Resins: Bulk Charge Transport and Interfacial Polarization Peaks. IEEE Trans. Dielectr. Electr. Insul. 2014, 21, 1330-1341. [CrossRef]

17. Gao, Y.; Liang, X.; Dissado, L.A.; Dodd, S.J.; Chalashkanov, N.M. Dielectric response of filled high temperature vulcanized silicone rubber. IEEE Trans. Dielectr. Electr. Insul. 2016, 23, 3683-3695. [CrossRef]

18. Wu, C.; Liang, X.; Dissado, L.A.; Chalashkanov, N.M.; Dodd, S.J.; Gao, Y.; Xu, S. Dielectric response of nano aluminium tri-hydrate filled silicone rubber. Compos. Sci. Technol. 2018, 163, 56-62. [CrossRef]

19. Dissado, L.A.; Hill, R.M. Anomalous low-frequency dispersion. Near direct current conductivity in disordered low-dimensional materials. J. Chem. Soc. Faraday Trans. 2 Mol. Chem. Phys. 1984, 80, 291-319. [CrossRef]

20. Tuncer, E.; Gubanski, S.M. Dielectric behavior of filled silicone rubbers: Effects of cross-linking agent and surface modified fillers. In Proceedings of the 2000 Annual Report Conference on Electrical Insulation and Dielectric Phenomena, Vicrotia, BC, Canada, 15-18 October 2000; pp. 175-178.

21. Kirst, K.; Kremer, F.; Litvinov, V. Broad-band dielectric spectroscopy on the molecular dynamics of bulk and adsorbed poly(dimethylsiloxane). Macromolecules 1993, 26, 975-980. [CrossRef]

22. Tuncer, E.; Gubanski, S.M. Electrical properties of filled silicone rubber. J. Phys. Condens. Matter 2000, 12, 1873-1897. [CrossRef]

23. Yuan, C.; Xie, C.; Li, L.; Xu, X.; Gubanski, S.M. Dielectric response characterization of in-service aged sheds of (U)HVDC silicone rubber composite insulators. IEEE Trans. Dielectr. Electr. Insul. 2016, 23, 1418-1426. [CrossRef]

24. Sillars, R.W. The properties of a dielectric containing semiconducting particles of various shapes. J. Inst. Electr. Eng. 1937, 12, 139-155.

25. Cai, J.J.; Salovey, R. Model filled rubber IV: Dependence of stress-strain relationship on filler particle morphology. J. Mater. Sci. 1999, 34, 4719-4726. [CrossRef]

26. Merckel, Y.; Diani, J.; Brieu, M.; Caillard, J. Effects of the amount of fillers and of the crosslink density on the mechanical behavior of carbon-black filled styrene butadiene rubbers. J. Appl. Polym. Sci. 2013, 129, 2086-2091. [CrossRef]

27. Polgar, L.M.; Hagting, E.; Raffa, P.; Mauri, M.; Simonutti, R.; Picchioni, F.; Duin, M. Effect of rubber polarity on cluster formation in rubbers cross-linked with diels-alder chemistry. Macromolecules 2017, 50, 8955-8964. [CrossRef] [PubMed]

28. Rubber, Vulcanized or Thermoplastic_Determination of Tensile Stress-Strain Properties; International Standard ISO 37: 2005(E); International Organization for Standardization (ISO): Geneva, Switzerland, 2005.

29. Vilgis, T.A.; Heienrich, G.; Kluppel, M. Reinforcement of Polymer Nano-Composites: Theory, Experiments and Applications; Cambridge University Press: Cambridge, UK, 2009.

30. Seiler, J.; Kindersberger, J. Insight into the Interphase in Polymer Nanocomposites. IEEE Trans. Dielectr. Electr. Insul. 2014, 21, 537-547. [CrossRef]

31. Jonscher, A.K. Dielectric Relaxation in Solids; Chelsea Dielectrics Press: London, UK, 1983.

32. Hill, R.M.; Dissado, L.A. The temperature dependence of relaxation processes. J. Phys. C Solid State Phys. 1982, 15, 5171-5193. [CrossRef]

33. Jonscher, A.K. Universal Relaxation Law; Chelsea Dielectrics Press: London, UK, 1996.

34. Andritsch, T.M. Epoxy Based Nanodielectrics for High Voltage DC-Applications—Synthesis, Dielectric Properties and Space Charge Dynamics. Ph.D. Thesis, Technische Universiteit Delft, Delft, The Netherlands, 2010.

35. Zou, C. The Effect of Humidity and Surface Functionalisation on the Dielectric Properties of Nanocomposites. Ph.D. Thesis, University of Leicester, Leicester, UK, 2007.

(C) 2019 by the authors. Licensee MDPI, Basel, Switzerland. This article is an open access article distributed under the terms and conditions of the Creative Commons Attribution (CC BY) license (http://creativecommons.org/licenses/by/4.0/). 